\title{
X-ray counterpart candidates for six new $\gamma$-ray pulsars
}

\author{
Dmitry A. Zyuzin, ${ }^{1 \star}$ Anna V. Karpova, ${ }^{1}$ and Yura A. Shibanov ${ }^{1}$ \\ ${ }^{1}$ Ioffe Institute, Politekhnicheskaya ul., 26, St. Petersburg, 194021, Russia
}

Accepted XXX. Received YYY; in original form ZZZ

\begin{abstract}
Using archival X-ray data we have found point-like X-ray counterpart candidates positionally coincident with six $\gamma$-ray pulsars discovered recently in the Fermi Gammaray Space Telescope data by the Einstein@Home project. The candidates for PSRs J0002+6216, J0554+3107, J1844-0346 and J1105-6037 are detected with Swift, and those for PSRs J0359+5414 and J2017+3625 are detected with Chandra. Despite a low count statistics for some candidates, assuming plausible constraints on the absorbing column density towards the pulsars, we show that X-ray spectral properties for all of them are consistent with those observed for other pulsars. J0359+5414 is the most reliably identified object. We detect a nebula around it, whose spectrum and extent suggest that this is a pulsar wind nebula powered by the pulsar. Associations of J0002+6216 and J1844-0346 with supernova remnants CTB 1 and G28.6-0.1 are proposed.
\end{abstract}

Key words: compact objects: general - neutron stars: individual (PSR J0002+6216, PSR J0359+5414, PSR J0554+3107, PSR J1105-6037, PSR J1844-0346, PSR $\mathrm{J} 2017+3625)$

\section{INTRODUCTION}

The Large Area Telescope (LAT) aboard the Fermi Gammaray Space Telescope has discovered over 200 new young and millisecond $\gamma$-ray pulsars ${ }^{1}$. The inferred parameters for the young pulsars characterise most of them as relatively energetic rotation powered nearby neutron stars (NSs). With spin-down powers $\dot{E} \gtrsim 10^{34} \mathrm{erg} \mathrm{s}^{-1}$ and characteristic ages $\tau_{c} \lesssim 1$ Myr they are likely located in less than a few kpc from the Sun (Abdo et al. 2013). Young NSs, in particular nearby ones, are rare (e.g., Noutsos et al. 2013). As such, these discoveries contribute significantly to more complete understanding of the pulsar population, NS physics, and birthrates (Watters \& Romani 2011). About a third of the LAT pulsars was detected during 'blind' searches for periodicity in sparse LAT $\gamma$-ray photons. Many of them remain undetected in the radio precluding independent distance estimates using the dispersion measure. In this case, follow-up observations at other wavelengths, especially in X-rays, were proven to be very productive (e.g., Marelli et al. 2013, 2015).

More distant and/or less energetic pulsars are weaker in $\gamma$-rays and long integration times are required for a detectable signal-to-noise ratio. The photon sparseness for them results in a large computational cost of the blind

\footnotetext{
^ E-mail: da.zyuzin@gmail.com (DAZ)

1 https://confluence.slac.stanford.edu/display/GLAMCOG/

Public+List+of+LAT-Detected+Gamma-Ray+Pulsars
}

search. The problem is solved with the aid of the volunteerbased computing power of the Einstein@Home project ${ }^{2}$ (Allen et al. 2013). Within this project, 19 new $\gamma$-ray pulsars have been recently discovered in the blind search of pulsations in about hundred of unidentified point-like LAT sources whose $\gamma$-ray spectra are similar to those of pulsars (Pletsch et al. 2013; Clark et al. 2015, 2016, 2017). All of them are isolated pulsars with $\tau_{c}$ between $3 \mathrm{kyr}$ and $2 \mathrm{Myr}$ and $\dot{E}$ between $10^{34}$ and $4 \times 10^{36} \mathrm{erg} \mathrm{s}^{-1}$. No radio pulsations have been reported so far for any of these new pulsars. Therefore, X-ray identification of these objects is crucial for their further study.

Using archival Swift and Suzaku data we have found a plausible X-ray counterpart and a possible pulsar wind nebula (PWN) for PSR J1932+1916, which is one of the youngest $\left(\tau_{c} \approx 35.4 \mathrm{kyr}\right)$ pulsars from the Einstein@Home sample (Karpova et al. 2017). The X-ray data allowed us to constrain the distance to the pulsar and to suggest its possible association with the supernova remnant (SNR) G54.40.3 .

Here we report the results of search for X-ray counterparts for the other eighteen Einstein@Home $\gamma$-ray pulsars using X-ray data archives and point source catalogues. We have found six counterpart candidates by position coincidence. We focus on their X-ray spectral properties and discuss if they are consistent with those of other Fermi pul-

\footnotetext{
2 http://einstein.phys.uwm.edu
} 
Table 1. X-ray counterpart candidates for six Fermi pulsars from the Einstein@Home sample. $\gamma$-ray coordinates are from Pletsch et al. (2013); Clark et al. (2017) and X-ray coordinates are from the 1st Swift X-ray Point Source Catalogue (1SXPS; Evans et al. 2014) and the Chandra Source Catalogue (Wang et al. 2016). Numbers in parentheses are $1 \sigma$ uncertainties for the $\gamma$-ray coordinates and 90 per cent confidence position errors for the X-ray counterpart candidates related to the last significant digits quoted. The last column shows significances of the X-ray candidate detections as the signal to noise ratio $S / N$.

\begin{tabular}{ccccccc}
\hline Fermi Pulsar & X-ray Counterpart Candidate & \multicolumn{1}{c}{$\mathrm{RA}_{\gamma}$} & \multicolumn{1}{c}{ Dec $_{\gamma}$} & $\mathrm{RAX}_{\mathrm{X}}$ & DecX & $S / N$ \\
\hline J0002+6216 & 1SXPS J000257.6+621609 & $00: 02: 58.17(2)$ & $+62: 16: 09.4(1)$ & $00: 02: 57.69(70)$ & $+62: 16: 09.2(4.9)$ & 1.8 \\
J0359+5414 & CXOGSG J035926.0+541455 & $03: 59: 26.01(2)$ & $+54: 14: 55.7(3)$ & $03: 59: 26.09(11)$ & $+54: 14: 55.8(1.0)$ & 23.8 \\
J0554+3107 & 1SXPS J055404.8+310741 & $05: 54: 05.01(3)$ & $+31: 07: 41(4)$ & $05: 54: 04.83(91)$ & $+31: 07: 41.7(6.2)$ & 2.7 \\
J1105-6037 & 1SXPS J110500.3-603713 & $11: 05: 00.48(4)$ & $-60: 37: 16.3(3)$ & $11: 05: 00.37(84)$ & $-60: 37: 13.1(6.4)$ & 2.0 \\
J1844-0346 & 1SXPS J184432.9-034626 & $18: 44: 32.89(2)$ & $-03: 46: 30.6(9)$ & $18: 44: 32.9(2)$ & $-03: 46: 26.6(2.7)$ & 5.9 \\
J2017+3625 & CXOGSG J201755.8+362507 & $20: 17: 55.84(1)$ & $36: 25: 07.9(2)$ & $20: 17: 55.81(8)$ & $36: 25: 07.8(1.0)$ & 5.5 \\
\hline
\end{tabular}

Table 2. Parameters of the pulsars listed in Table 1, which are derived from the Fermi-LAT timing solutions Pletsch et al. (2013); Clark et al. (2017): a period $P$, a period derivative $\dot{P}$, a characteristic age $\tau_{c}=P / 2 \dot{P}$, a spin-down luminosity $\dot{E}$, a dipole magnetic field $B$. The Fermi-LAT flux density $G_{100}$ in the $100 \mathrm{MeV}-100 \mathrm{GeV}$ range is taken from 3FGL catalogue (Acero et al. 2015). $d_{\gamma}$ is a pseudo-distance (see text for details).

\begin{tabular}{cccccccc}
\hline Fermi Pulsar & $\begin{array}{c}P \\
(\mathrm{~ms})\end{array}$ & $\begin{array}{c}\dot{P} \\
\left(10^{-15} \mathrm{~s} \mathrm{~s}^{-1}\right)\end{array}$ & $\begin{array}{c}\tau_{\mathrm{c}} \\
(\mathrm{kyr})\end{array}$ & $\begin{array}{c}\dot{E} \\
\left(10^{33} \mathrm{erg} \mathrm{s}^{-1}\right)\end{array}$ & $\begin{array}{c}B \\
\left(10^{12} \mathrm{G}\right)\end{array}$ & $\begin{array}{c}G_{100} \\
\left(10^{-11} \mathrm{erg} \mathrm{cm}^{-2} \mathrm{~s}^{-1}\right)\end{array}$ & $\begin{array}{c}d_{\gamma} \\
(\mathrm{kpc})\end{array}$ \\
\hline J0002+6216 & 115.4 & 5.97 & 306 & 153 & 0.8 & 1.83 & 2.3 \\
$\mathrm{~J} 0359+5414$ & 79.4 & 16.7 & 75 & 1318 & 1.2 & 2.45 & 3.45 \\
$\mathrm{~J} 0554+3107$ & 465.0 & 142.6 & 52 & 56 & 8.2 & 1.73 & 3.9 \\
$\mathrm{~J} 1105-6037$ & 194.94 & 21.8 & 141 & 116 & 2.1 & 2.8 & 1.53 \\
$\mathrm{~J} 1844-0346$ & 112.85 & 154.7 & 12 & 4249 & 4.2 & 6.5 & 0.656 \\
$\mathrm{~J} 2017+3625$ & 166.75 & 1.36 & 1943 & 12 & 0.5 & & \\
\hline
\end{tabular}

sars which have been firmly identified in X-rays. Possible associations with nearby SNRs are proposed for two pulsars which allow for independent distance estimates. In Sect. 2 we present the search results and describe the general techniques and tools used for the X-ray analysis of the counterpart candidates. The X-ray properties of each candidate and the possible SNR associations are thoroughly considered in sub-sections of Sect. 3. Background optical objects within the position error ellipses of the candidates are considered in Sect. 4. In Sect. 5, we discuss the results.

\section{X-RAY COUNTERPART SEARCH RESULTS}

Using X-ray catalogues ${ }^{3}$, we have found point-like Xray counterpart candidates for six out of eighteen Einstein@Home $\gamma$-ray pulsars, which coincided by position with the Fermi pulsars at $90 \%$ confidence level. Four candidates have been detected with Swift X-Ray Telescope (XRT) and the other two have been detected with Chandra Advanced CCD Imaging Spectrometer (ACIS). The Fermi pulsar and X-ray counterpart candidate catalogue names and respective $\gamma$ - and X-ray coordinates with their uncertainties are presented in Table 1 . The parameters of the pulsars derived from the $\gamma$-ray data are shown in Table 2 . For radio-quiet pulsars the distances are estimated using the empirical 'pseudo'-distance relation for $\gamma$-ray pulsars (Saz Parkinson et al. 2010): $d_{\gamma}=1.6 \times\left(\dot{E}\left[10^{34} \mathrm{erg} \mathrm{s}^{-1}\right]\right)^{0.25} \times$

3 https://heasarc.gsfc.nasa.gov/w3browse/all/xray.html; http://www.swift.ac.uk/1SXPS/
$\left(G_{100}\left[10^{-11} \mathrm{erg} \mathrm{cm}^{-2} \mathrm{~s}^{-1}\right]\right)^{-0.5} \mathrm{kpc}$, where $G_{100}$ is the pulsar flux density in the $100 \mathrm{MeV}-100 \mathrm{GeV}$ range measured with the Fermi-LAT.

In most cases, the regions containing the pulsars were observed in X-rays several times. For Swift candidates, the number of observations varies from 2, for J0554+3107, to 64, for J1844-0346, and the total exposure times varies from 9.2 , for J0002+6216, to $100 \mathrm{ks}$, for J1844-0346, respectively. For Chandra candidates, they varied from 1 to 9 and from $10 \mathrm{ks}$ to $460 \mathrm{ks}$, for $\mathrm{J} 2017+3625^{4}$ and J0359+5414 ${ }^{5}$, respectively. In Table 1, the coordinates of the Swift candidates are taken from the 1st Swift X-ray Point Source Catalogue (Evans et al. 2014). They were measured on the stacked XRT images. For Chandra objects, the archival ACIS data were reprocessed using the CIAO v.4.9 tool CHANDRA_REPRO. For multiple observations, the data were merged using the MERGE_OBS task. The candidate coordinates were measured on the resulting images using the WAVDETECT tool. They are consistent with those provided by the Chandra Source Catalogue (Wang et al. 2016).

The sections of the stacked/merged X-ray images containing the pulsars are presented in Fig. 1 where dashed yellow and solid white ellipses show $1 \sigma$ position uncertainties of the $\gamma$-ray pulsars and centroids of their X-ray counterpart candidates, respectively. The former account for the Fermi $1 \sigma$ timing position uncertainties from Table 1 and

\footnotetext{
${ }^{4}$ Observation ID 14699

5 Observation IDs 4657, 14688, 14689, 14690, 15548, 15549, $15550,15585,15586$
} 

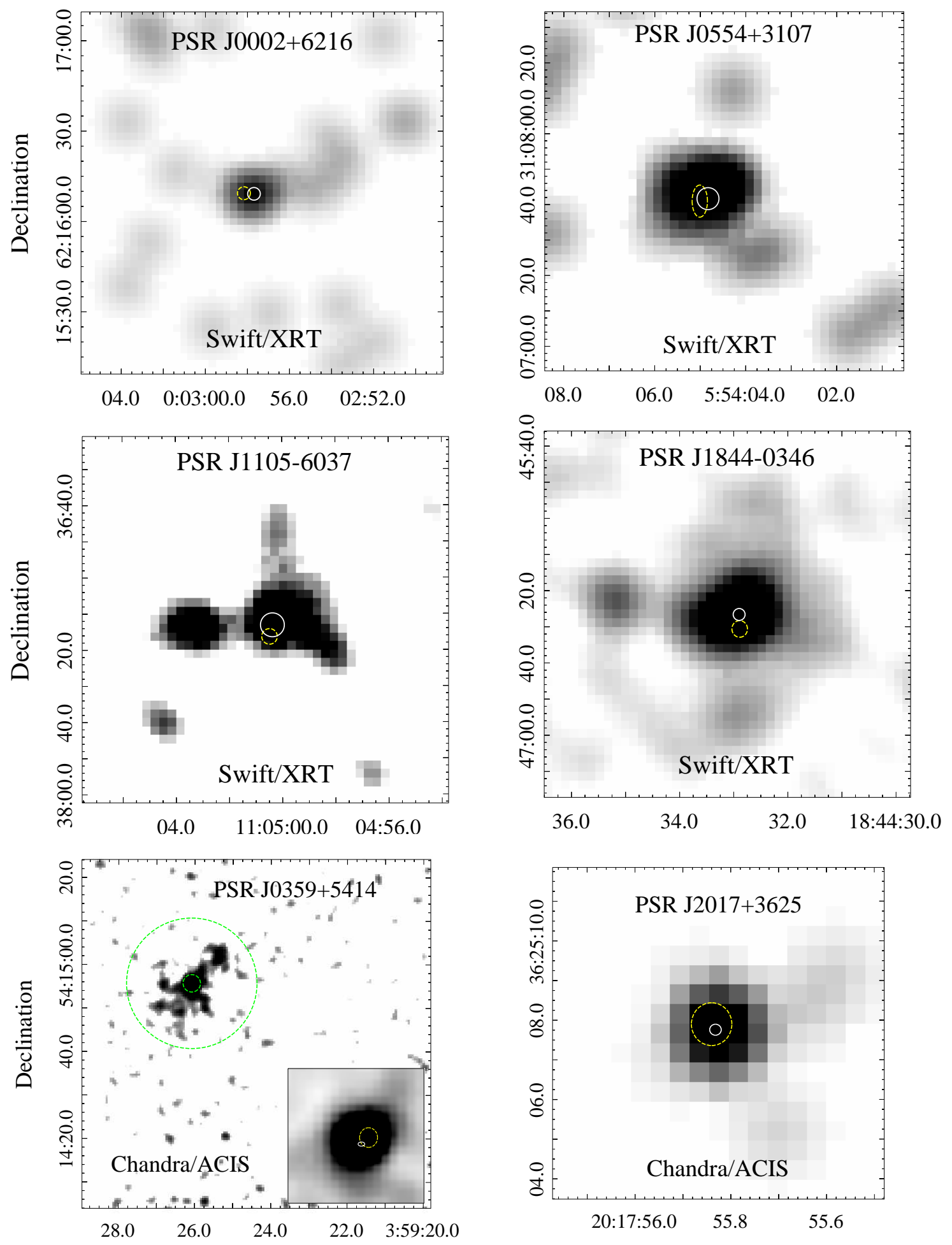

Right ascension

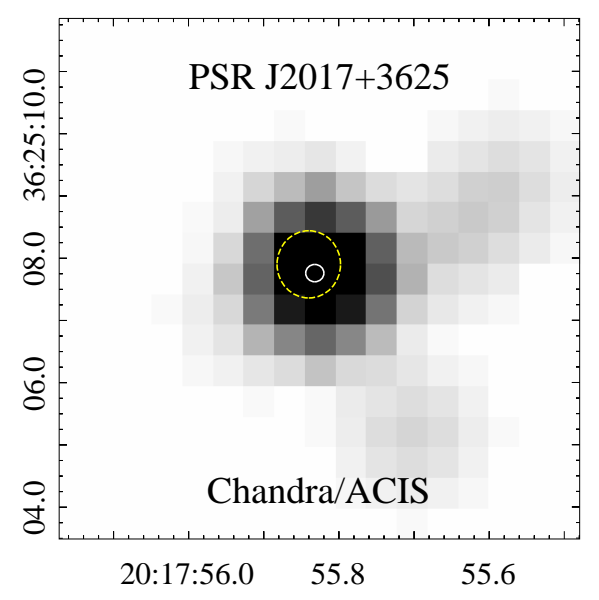

Right ascension

Figure 1. Swift/XRT and Chandra/ACIS images of the point-like X-ray counterpart candidates for the $\gamma$-ray pulsars listed in Table 1. $1 \sigma$ positional uncertainties of the point-like candidate centroids and the $\gamma$-ray pulsars in the images are shown by solid white and dashed yellow ellipses, respectively. The green annulus in the bottom-left panel was used to extract the spectrum of the extended emission around the point-like counterpart candidate. The insert in this panel shows the zoomed-in 8 arcesec $\times 8$ arcsec region with the candidate near the centre of the extended emission. A different count scale is used in the insert to reveal the point-like counterpart candidate. 
the X-ray telescope $1 \sigma$ astrometric accuracy. This quantity, in turn, is estimated from $90 \%$ confidence aspect solution accuracy of 3.' 5 and 0.' 8 for Swift (Evans et al. 2014) and $\mathrm{Chandra}^{6}$, respectively. The solid white ellipses include only statistical point source position measurement errors on the stacked/merged images. It is seen that positions of the counterpart candidates are consistent with those of the pulsars in all cases.

Due to shallow total exposures, mainly of about $10 \mathrm{ks}$, the formal X-ray signal-to-noise ratio $S / N$ for the counterpart candidates on the XRT images is low. It varies from 1.8, for J0002+6216, to 5.9, for J1844-0346 (see the last column in Table 1). Such a low $S / N$ is the main reason for relatively large absolute position errors as compared to the Fermi position errors (cf. Table 1). Nevertheless, all the objects are classified as 'Good' point sources in the Swift catalogue based on an iterative point spread function and likelihood analysis (Evans et al. 2014). $S / N$ for the ACIS counterpart candidates of $\mathrm{J} 2017+3625$ and $\mathrm{J} 0359+5414$ is $\approx 5.5$ and $\approx 23.8$, respectively.

As one can see in Fig. 1, there is a diffuse emission around the point-like candidate of J0359+5414 extended roughly along SE-NW direction by about 30 arcsec. This can be the PWN, which is not a surprise for the pulsar with a relatively high spin-down power of $\approx 10^{36} \mathrm{erg} \mathrm{s}^{-1}$. The visible size of the presumed PWN $(\approx 30 \operatorname{arcsec} \times 20$ arcsec $)$ is roughly compatible with that of the Vela PWN $(\approx 6$ arcmin $\times 5.5$ arcmin) assuming that the latter is moved to about 12 times larger distance corresponding to J0359+5414 (from 0.3 to $3.5 \mathrm{kpc}$ ).

For further spectral analysis of the proposed counterpart candidates detected with Swift, we have obtained their spectra using the Swift/XRT data product generator (Evans et al. 2009). For two Chandra candidates, the spectra were extracted from the archival data using the CIAO SPECEXTRACT tool. For the J0359+5414 candidate, we have used the extraction aperture with a 2 arcsec radius for the point-like source, whereas for the extended source, we have used the annulus centred at the point-like candidate with the inner and outer radii of 2 and 15 arcsec, respectively. The annulus is shown in Fig. 1. For the J2017+3625 candidate, a 1.5 arcsec aperture radius was used. In all the cases, the background was taken from regions free of any sources.

The spectra were fitted in the $0.3-10 \mathrm{keV}$ range using the XSPEC v.12.9.0 tool by plausible absorbed spectral models. We applied a power law (PL) model, describing nonthermal radiation from the NS magnetosphere, a blackbody (BB) model and a magnetised NS atmosphere models NSMAXG (Ho et al. 2008), describing the thermal emission from the NS surface. Depending on the count statistics, we used either a single model or a composite model. The latter is the sum of the thermal and nonthermal spectral components, e.g. BB+PL or NSMAXG+PL. For the diffusive source, we applied the PL model assuming the synchrotron nature of the PWN emission. For the photoelectric absorption, the tbabs model with the wilm interstellar abundances was used. Due to low count numbers $C$-statistics (Cash 1979) was used to estimate fit qualities. Unless stated otherwise, all the spectra were binned by at least 1 count per energy bin.

6 cxc.harvard.edu/cal/ASPECT/almen

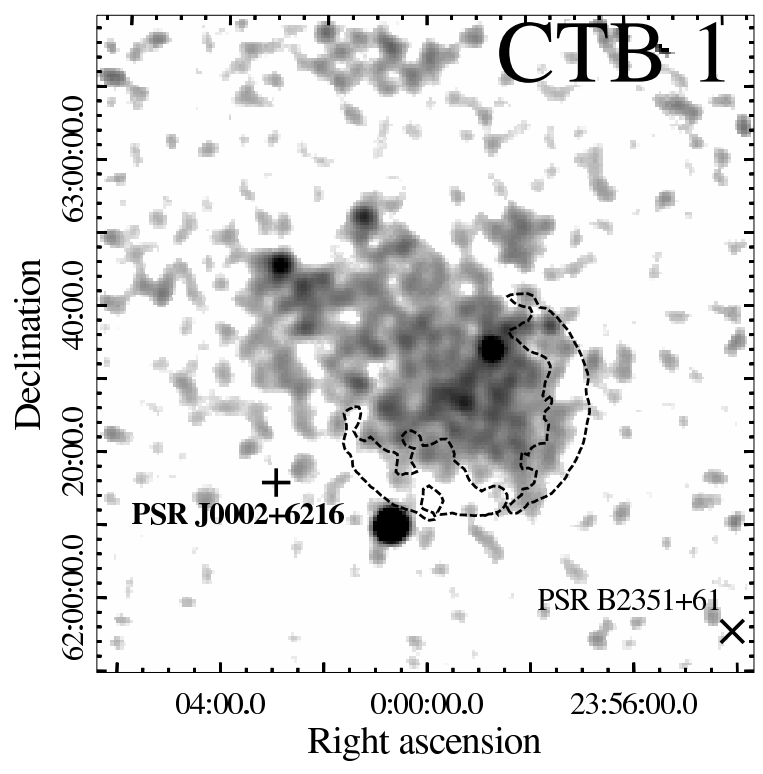

Figure 2. ROSAT X-ray image of the field containing the SNR CTB 1, PSR J0002+6216, and unrelated PSR B2351+61 whose positions are marked by a cross and an ' $\mathrm{X}$ ', respectively. Contours show the CTB 1 radio shell structure obtained from the Westerbork Northern Sky Survey (WENSS; Rengelink et al. 1997).

Independent information on the absorbing column density $\mathrm{N}_{H}$ towards a pulsar was employed, when this was required to facilitate the fit.

\section{PROPERTIES OF THE PULSAR X-RAY COUNTERPART CANDIDATES}

\section{$3.1 \quad$ PSR J0002+6216}

The counterpart candidate for this pulsar accounts for only 9 XRT source counts detected during the $\approx 9.2 \mathrm{ks}$ total exposure. This precludes any quantitative spectral analysis.

$N_{\mathrm{H}}$ can be estimated if the pulsar is associated with the well studied SNR CTB 1 (G116.9+0.2). The ROSAT X-ray image of CTB 1 is presented in Fig. 2. This is an oxygen-rich mixed morphology core-collapse remnant where a black-hole or an NS are expected to be born at the supernova event but none have been found so far (Pannuti et al. 2010a,b). As seen from Fig. 2, PSR J0002+6216 is located near the edge of the remnant at the angular distance of only about 17 arcmin from its centre. Another nearby pulsar B2351+61 shown in the image cannot be associated with CTB 1 because its trail calculated using known proper motion and characteristic age (Harrison et al. 1993) does not point to the remnant. The PSR J0002+6216 pseudo-distance (Table 2) is consistent with the SNR distance range of 1.5-4 kpc (e.g., Fesen et al. 1997) favouring the association.

The age of CTB 1 is estimated to be in the range of 7.5-44 kyr (Koo \& Heiles 1991; Hailey \& Craig 1994). This is smaller than the pulsar characteristic age of 306 kyr. However, such a large discrepancy is frequently observed for pulsars associated with SNRs (e.g., PSR J0538+2817 in the SNR S147 (Kramer et al. 2003); PSR J1101-6101 in the 
SNR MSH 11-61A (Halpern et al. 2014)). If the real pulsar age is consistent with that of the SNR, then the initial pulsar period $P_{0}$ according to the standard real age $t-P \dot{P}$ relation,

$$
t=\frac{P}{(n-1) \dot{P}}\left[1-\left(\frac{P_{0}}{P}\right)^{n-1}\right],
$$

was in the range of 107-114 ms for the magnetic dipole spindown model with $n=3$. This is a typical value for birth periods (see, e.g., Popov \& Turolla 2012; Noutsos et al. 2013) which is also in a good agreement with recent core-collapse models (e.g., Wongwathanarat et al. 2013; Fuller et al. 2015).

Based on the 17 arcmin pulsar offset from the CTB 1 centre, its expected proper motion and transverse velocity are $\mu \approx 50 t_{20}^{-1}$ mas $\mathrm{yr}^{-1}$ and $v_{\perp} \approx 550 d_{2.3} t_{20}^{-1} \mathrm{~km} \mathrm{~s}^{-1}$, where $d_{2.3}$ is the pulsar distance scaled to its pseudo-distance of $2.3 \mathrm{kpc}$ (Table 2) and $t_{20}$ is the real age divided by $20 \mathrm{kyr}$. Given the age uncertainty of 7.5-44 kyr, the $v_{\perp}$ uncertainty of $(250-1500) d_{2.3} \mathrm{~km} \mathrm{~s}^{-1}$ is large, but it is compatible with the mean 2D pulsar speed of $\approx 250 \mathrm{~km} \mathrm{~s}^{-1}$ (Hobbs et al. 2005 ) and the largest NS velocity of $\approx 1100 \mathrm{~km} \mathrm{~s}^{-1}$ precisely measured so far (Chatterjee et al. 2005).

Therefore, the association of PSR J0002+6216 and CTB 1 appears to be plausible. Assuming that it is real, we fixed $N_{\mathrm{H}}$ for the pulsar at $6 \times 10^{21} \mathrm{~cm}^{-2}$, the value accepted for the remnant (Pannuti et al. 2010a). Then, to estimate the pulsar counterpart candidate flux, we fitted its spectrum with the absorbed PL with the photon index $\Gamma=2$, which is typical for pulsars (e.g., Kargaltsev \& Pavlov 2008). The resulting $C$-value was 12 per 6 degrees of freedom (d.o.f.). The fit resulted in the observed and unabsorbed X-ray fluxes of $4.4_{-2.0}^{+2.8} \times 10^{-14}$ and $7.4_{-3.9}^{+4.6} \times 10^{-14}$ erg $\mathrm{cm}^{-2} \mathrm{~s}^{-1}$, respectively ${ }^{7}$, in the $0.3-10 \mathrm{keV}$ band. For the distance of $2.3 \mathrm{kpc}$ this translates into the X-ray luminosity of $4.7_{-2.5}^{+2.9} \times 10^{31} d_{2.3}^{2} \mathrm{erg} \mathrm{s}^{-1}$. It is consistent with nonthermal X-ray luminosities of 10-100 kyr old pulsars (e.g., Kargaltsev \& Pavlov 2008). The X-ray efficiency in the 0.3$10 \mathrm{keV}$ range is $\log \left(L_{\mathrm{X}} / \dot{E}\right) \approx-3.5$. The ratio between the $\gamma$-ray and unabsorbed X-ray fluxes is $\log \left(G_{100} / F_{X}\right) \approx 2.4$. According to Abdo et al. (2013, see their Fig. 18), this is consistent with a typical for radio-loud $\gamma$-ray pulsars ratio of $2.4 \pm 1.1$, encouraging further search for the pulsar radio counterpart ${ }^{8}$.

Alternatively, the single BB fit results in the temperature $T=147_{-43}^{+84} \mathrm{eV}$, the emitting area radius $R=1.3_{-1.2}^{+3.3} d_{2.3}$ $\mathrm{km}$, and the observed and unabsorbed X-ray fluxes of $1.5_{-0.6}^{+0.8} \times 10^{-14}$ and $1.2_{-0.8}^{+2.4} \times 10^{-13} \mathrm{erg} \mathrm{cm}^{-2} \mathrm{~s}^{-1}$, respectively. The fit quality was only marginally better than that for the PL model, $C$ (d.o.f $)=9.6(5)$. In principle, this result could be consistent with the thermal emission from pulsar hot polar caps. However, uncertainties are too large for definite conclusions.

To summarise, we can state that the detected X-ray source is a plausible counterpart of PSR J0002+6216.

7 Hereafter, $1 \sigma$ flux and fitting parameter errors are presented. 8 After this paper has been submitted, the detection of J0002+6216 in the radio was reported (Wu et al. 2017).

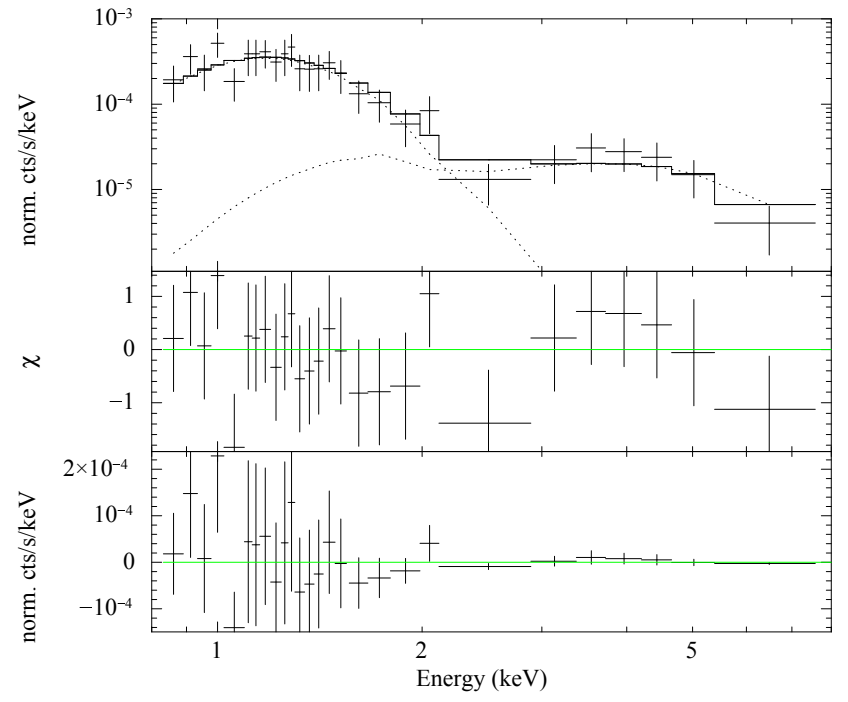

Figure 3. Top panel: X-ray spectrum of the PSR J0359+5414 X-ray counterpart candidate best fitted by the sum (solid line) of the NSMAXG and the PL models which are dominated in the 0.5-2 and 2-8 keV ranges, respectively (dotted lines). Middle and bottom panels: fit residuals.

\section{$3.2 \quad$ PSR J0359+5414}

To reveal the nature of the serendipitously detected Chandra pulsar and PWN counterpart candidates, we have used the COMBINE_SPECTRA tool to co-add the source and background spectra from all the observation IDs. This resulted in 150 and 293 ACIS source counts for the point and the diffuse objects, respectively, detected during the $\approx 460 \mathrm{ks}$ total exposure in the $0.3-10 \mathrm{keV}$ range. The spectra were grouped to ensure 5 and 10 counts per the energy bin for the presumed pulsar and PWN, respectively. We fitted the spectra simultaneously with the shared $N_{\mathrm{H}}$ parameter using all spectral models listed in Sect. 2.

For the pulsar counterpart candidate only the composite models $\mathrm{BB}+\mathrm{PL}$ and NSMAXG $+\mathrm{PL}$ were statistically acceptable. Both of them provided equal fit qualities, $C_{\mathrm{BB}+\mathrm{PL}}($ d.o.f. $)=89.8(85)$ and $C_{\mathrm{NSMAXG}+\mathrm{PL}}($ d.o.f. $)=$ 89.95(86). For the PL+NSMAXG model (with NS mass $M_{\mathrm{NS}}=1.4 \mathrm{M}_{\odot}$, radius $R_{\mathrm{NS}}=13 \mathrm{~km}$ and magnetic field $B=10^{12} \mathrm{G}$ ), fixing the distance at the pseudo-distance value of $3.45 \mathrm{kpc}$, we have obtained the column density $N_{\mathrm{H}}=(0.9 \pm 0.2) \times 10^{22} \mathrm{~cm}^{-2}$, the photon index $\Gamma=0.5 \pm 0.6$ and the NS temperature (gravitationally redshifted) $T^{\infty}=$ $(6.1 \pm 0.2) \times 10^{5} \mathrm{~K}(\approx 53 \mathrm{eV})$. For the $\mathrm{BB}+\mathrm{PL}$ model, the column density $N_{\mathrm{H}}=(0.9 \pm 0.3) \times 10^{22} \mathrm{~cm}^{-2}$, the photon index $\Gamma=0.8 \pm 0.6$, the temperature $T=162_{-25}^{+29} \mathrm{eV}$ and the emitting area radius $R=0.8_{-0.4}^{+1.1} d_{3.45} \mathrm{~km}$, where $d_{3.45}$ is the pulsar distance normalised to the pseudo-distance. In this case, the thermal emission comes from a compact hot region on the NS surface whose radius is consistent with the polar cap radius of $0.76 \mathrm{~km}$ estimated for this pulsar in the standard way (e.g., Sturrock 1971). For the presumed PWN, in both cases we got $\Gamma=1.6 \pm 0.3$, which is typical for PWNe (Kargaltsev \& Pavlov 2008). For the NSMAXG+PL case, the point-like and the extended object spectra and the respective best fit models are presented in Figs. 3 and 4.

Our spectral analysis strongly supports the pulsar na- 


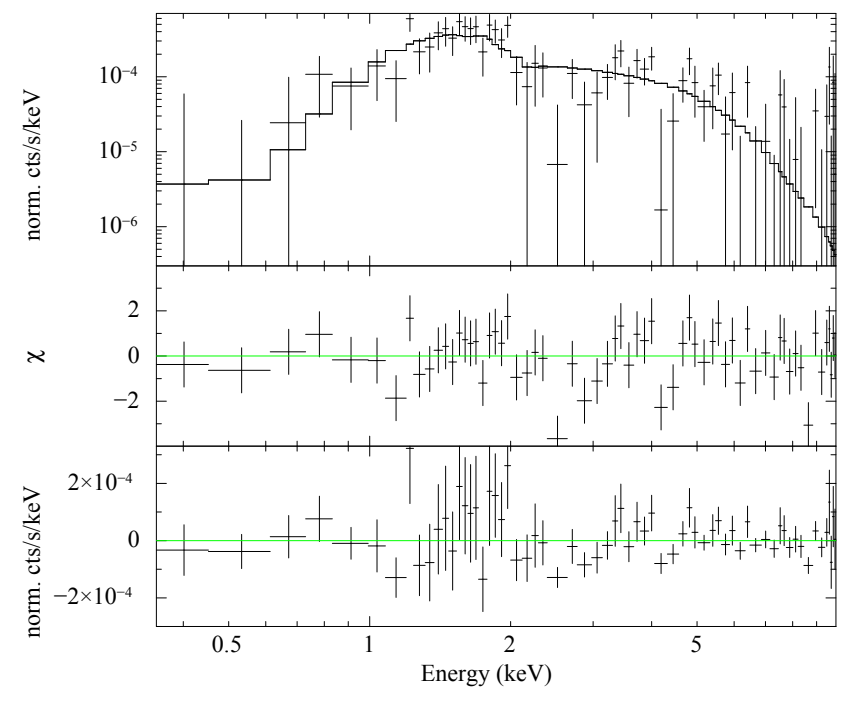

Figure 4. Top panel: X-ray spectrum of the presumed PWN of PSR J0359+5414 and the best-fit PL model (solid line). Middle and bottom panels: fit residuals.

ture of the counterpart candidate. Its X-ray spectrum is consistent with the spectrum expected from this middle-aged pulsar with the soft thermal component from the entire surface of the cooling NS, or from its hot regions, and a hard nonthermal spectral tail of the pulsar magnetosphere origin.

The absorbing column density value for PSR J0359+5414 derived from the spectral fit is consistent with $N_{\mathrm{H}} \approx 1.1 \times 10^{22} \mathrm{~cm}^{-2}$ estimated towards the pulsar for the pseudo-distance of $3.45 \mathrm{kpc}$ using the relation $N_{\mathrm{H}}=(8.9 \pm 0.4) \times E(B-V) \times 10^{21} \mathrm{~cm}^{-2}$ (Foight et al. 2016) and the interstellar extinction $E(B-V)$-distance relation which is based on the Pan-STARRS and 2MASS photometry $^{9}$ (Green et al. 2015). This means that $3.45 \mathrm{kpc}$ is a quite realistic distance estimate.

For the pulsar and PWN spectra, the unabsorbed nonthermal fluxes in the $0.3-10 \mathrm{keV}$ range are $(9 \pm 3) \times 10^{-15}$ and $(2 \pm 0.3) \times 10^{-14} \mathrm{erg} \mathrm{cm}^{-2} \mathrm{~s}^{-1}$, respectively. These translate into nonthermal luminosities $L_{\mathrm{X}}^{\mathrm{psr}} \approx 1.3 \times 10^{31} d_{3.45}^{2}$ and $L_{\mathrm{X}}^{\mathrm{pwn}} \approx 2.8 \times 10^{31} d_{3.45}^{2} \mathrm{erg} \mathrm{s}^{-1}$ which are compatible with known PSR/PWN luminosities of pulsars with $\dot{E} \sim 10^{36}$ $\mathrm{erg} \mathrm{s}^{-1}$ (Kargaltsev \& Pavlov 2008). Finally, the ratio between the $\gamma$-ray and the nonthermal unabsorbed $\mathrm{X}$-ray fluxes of the pulsar is $\log \left(G_{100} / F_{\mathrm{X}}\right) \approx 3.4$. This is consistent with the value of $3.5 \pm 0.5$ derived for the radio-quiet population of $\gamma$-ray pulsars (Abdo et al. 2013).

To summarise, the X-ray properties of the Chandra point and extended sources leave little doubts that they represent the real X-ray counterparts of PSR J0359+5414 and its PWN.

\section{$3.3 \quad$ PSR J0554+3107}

There are 17 source counts from the XRT counterpart candidate detected during the $\approx 10 \mathrm{ks}$ total exposure in the $0.3-10$ $\mathrm{keV}$ range. This precludes any quantitative spectral analysis

9 http://argonaut.skymaps.info/ using the XRT data alone. Nevertheless, since 13 out of 17 counts are detected in the $0.3-1 \mathrm{keV}$ band, a preliminary conclusion can be drown that the source spectrum is soft. This suggests that the detected emission may be dominated by the thermal component from the NS surface, as is typically observed for middle-aged pulsars, a class to which PSR J0554+3107 likely belongs in view of its characteristic age (Table 2).

This is further supported by the pulsar association with an evolved SNR G179.0+2.6 proposed by Pletsch et al. (2013). The pulsar characteristic age of $52 \mathrm{kyr}$ is compatible with the remnant age range of 10-100 kyr (Fuerst \& Reich 1986). Detection of G179.0+2.6 in the optical O III narrow band ${ }^{10}$ implies that this is an oxygen rich core-collapse SNR where a NS could have been born. Based on an empirical surface luminosity-SNR angular size relation (Milne 1979) and $d_{\gamma}$ for the pulsar (Table 2), the likely distance to the presumed pulsar+SNR system lies in the range of 1.9-3.5 $\mathrm{kpc}$.

This distance range, the $E(B-V)$-distance (Green et al. $2015)$ and $E(B-V)-N_{\mathrm{H}}$ (Foight et al. 2016) relations allow us to constrain $N_{\mathrm{H}}$ for the pulsar to the range of $(1.9-2.5) \times 10^{21}$ $\mathrm{cm}^{-2}$ and perform a rough spectral analysis of the counterpart candidate. Fixing $N_{\mathrm{H}}$ in the obtained range we have fitted the XRT source spectrum in the $0.3-10 \mathrm{keV}$ range by the PL, BB, and NSMAX models. For the latter model, we have selected $B=10^{13} \mathrm{G}$ and the gravitational redshift parameter $1+z_{g}=1.21$, corresponding to $M_{\mathrm{NS}}=1.4 \mathrm{M}_{\odot}$ and $R_{\mathrm{NS}}=13 \mathrm{~km}$.

The PL fit results in an unreasonably steep spectral slope with $\Gamma>4$. This is not typical for pulsars and thus can be rejected. Both thermal fits are equally acceptable with $C$ (d.o.f. $)=15(12)$. The $\mathrm{BB}$ fit results in the temperature of $100-110 \mathrm{eV}$ and the emitting area radius of $(2-3) d_{1.9} \mathrm{~km}$ depending on the $N_{\mathrm{H}}$ value from the allowed range. Here $d_{1.9}$ is the pulsar distance in units of $1.9 \mathrm{kpc}$. The NSMAX fit yields the NS surface temperature and emitting area radius (gravitationally redshifted) of $47-54 \mathrm{eV}$ and (11-19) $d_{1.9} \mathrm{~km}$, respectively. For both thermal models the absorbed flux is about $(3.1-3.2) \times 10^{-14} \mathrm{erg} \mathrm{cm}^{2} \mathrm{~s}^{-1}$ in the $0.3-10 \mathrm{keV}$ band.

The temperature and the emitting area derived from the fits using the thermal emission models are consistent with those expected for a middle-aged cooling NS. This supports the pulsar nature of the XRT counterpart candidate.

\section{$3.4 \quad$ PSR J1105-6037}

There are 16 source counts from the XRT counterpart candidate detected during the $\approx 16 \mathrm{ks}$ total exposure in the $0.3-10$ $\mathrm{keV}$ range. 10 out of 16 counts are in $0.3-1 \mathrm{keV}$ range implying, as in the case of PSR J0554+3107, that the spectrum may contain a soft thermal component corresponding to the surface emission from a middle-aged NS.

Unfortunately, no advanced interstellar extinctiondistance relation is provided for the pulsar direction by Green et al. (2015) to constrain $N_{\mathrm{H}}$ to the pulsar and to facilitate spectral fits. Therefore we have utilised the extinction map which is based on a model fit to the COBE DIRBE

10 http://astro.neutral.org/imagehtml/20161128-snrG179.0+2.6.html 


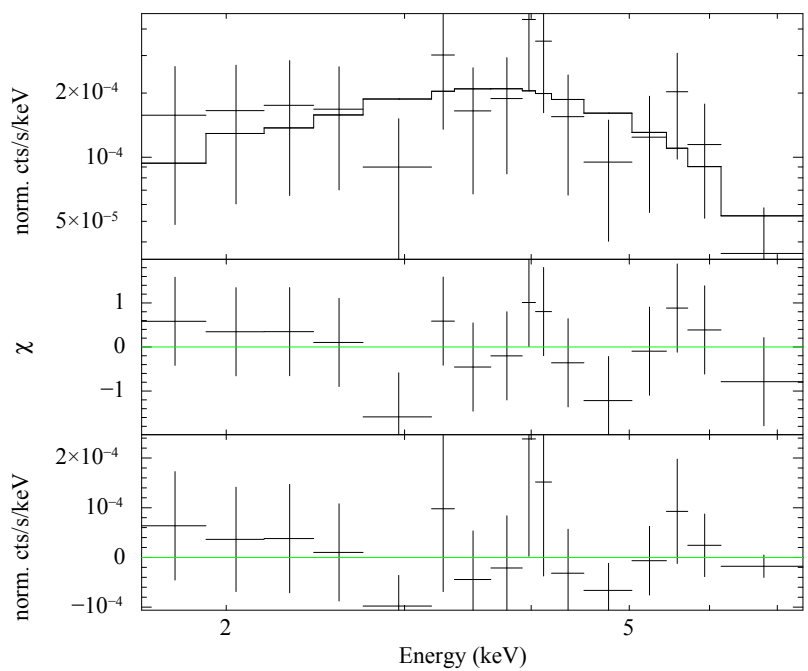

Figure 5. Top panel: X-ray spectrum of the PSR J1844-0346 Xray counterpart candidate and the best-fit PL model. The spectrum is binned to ensure at least 4 counts per energy bin for illustration purposes only. Middle and bottom panels: residuals

data (Drimmel et al. 2003). Applying the $E(B-V)-N_{\mathrm{H}}$ relation (Foight et al. 2016) we obtained $N_{\mathrm{H}} \approx 3.2 \times 10^{21} \mathrm{~cm}^{-2}$ for the pulsar pseudo-distance of $1.53 \mathrm{kpc}$ (Table 2).

Fixing $N_{\mathrm{H}}$ at this value, we have fitted the source spectrum trying the PL, BB and NSMAXG models. The PL fit resulted in a too steep photon index $\Gamma \approx 4$ which is not typical for pulsars. The nonthermal model can thus be rejected. Both thermal models resulted in acceptable fits with $C_{\mathrm{BB}}($ d.o.f. $)=5.7(9)$ and $C_{\mathrm{NSMAXG}}($ d.o.f. $)=6.3(10)$. Using the $\mathrm{BB}$ model, we obtain the temperature of $165_{-38}^{+58} \mathrm{eV}$ and the radius of the emitting area of $0.5_{-0.3}^{+0.6} d_{1.53} \mathrm{~km}$, where $d_{1.53}$ is the pulsar distance in units of the pseudo-distance. The latter value is consistent with the polar cap radius of $0.49 \mathrm{~km}$ estimated for this pulsar in the standard way (e.g., Sturrock 1971). For the NSMAXG model (the NS mass $M_{\mathrm{NS}}=1.4 \mathrm{M}_{\odot}$, radius $R_{\mathrm{NS}}=13 \mathrm{~km}$ and magnetic field $B=10^{12} \mathrm{G}$ ), fixing the distance at the pseudo-distance value, we obtain the gravitationally redshifted temperature of $(5.1 \pm 0.3) \times 10^{5} \mathrm{~K}$ $(\approx 44 \mathrm{eV})$. The observed flux in the $0.3-10 \mathrm{keV}$ band is $\approx 2 \times 10^{-14} \mathrm{erg} \mathrm{cm}^{2} \mathrm{~s}^{-1}$ for both thermal models.

The thermal fit parameters are reasonable for a middleaged cooling NS supporting the pulsar nature of the XRT candidate counterpart.

\subsection{PSR J1844-0346}

This is the youngest and most energetic pulsar in our sample but it is likely to be the most distant object as well (Table 2). About 100 source counts during the $\approx 100 \mathrm{ks}$ total exposure were detected from the XRT candidate allowing one to perform an initial spectral analysis without additional assumptions. The spectrum is well fitted by the PL model with $N_{\mathrm{H}}=5_{-3}^{+4} \times 10^{22} \mathrm{~cm}^{-2}$ and $\Gamma=0.9_{-0.8}^{+0.9} ; C_{\mathrm{PL}}$ (d.o.f.) $=44.2(61)$. This suggests that the candidate can be indeed a distant young pulsar whose spectrum is dominated by the nonthermal emission of the NS magnetosphere origin, as is observed, e.g., for the Crab pulsar. The BB fit is also acceptable but results in an unrealistically high for NSs temperature $>$

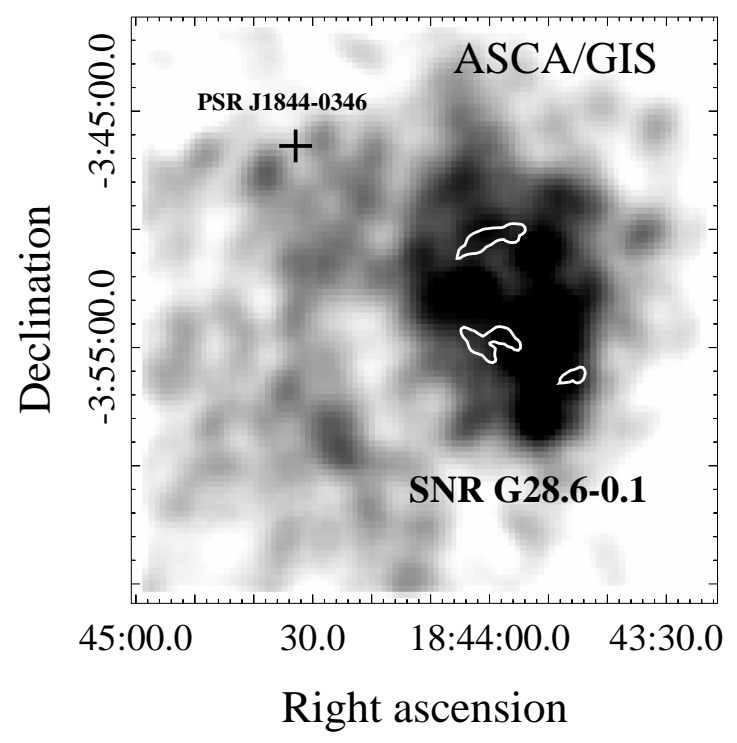

Figure 6. $A S C A /$ GIS X-ray image of the field containing the SNR G28.6-0.1 and PSR J1844-0346 whose position is marked by a cross. Contours show the G28.6-0.1 radio structure obtained from the MAGPIS (The Multi-Array Galactic Plane Imaging Survey) VLA $1.4 \mathrm{GHz}$ data (Helfand et al. 2006).

$1.5 \mathrm{keV}$. Due to this reason, the thermal model can be rejected. The spectrum with the PL fit is shown in Fig. 5. The observed and unabsorbed fluxes are $1.6_{-0.4}^{+0.5} \times 10^{-13}$ and $2.2_{-0.4}^{+1.3} \times 10^{-13} \mathrm{erg} \mathrm{cm}^{-2} \mathrm{~s}^{-1}$, respectively, in the $0.3-10 \mathrm{keV}$ band. This yields the X-ray luminosity of $4.9_{-0.9}^{+2.9} \times 10^{32} d_{4.3}^{2}$ erg s$~^{-1}$, where $d_{4.3}$ is the pulsar distance in units of the pseudo-distance. $\log \left(G_{100} / F_{\mathrm{X}}\right) \approx 2.1$ is compatible with the value obtained for the radio-loud $\gamma$-ray pulsar population (Abdo et al. 2013).

PSR J1844-0346 is located about 10 arcmin off the G28.6-0.1 SNR centre (Fig. 6). This is a young SNR thoroughly explored by Ueno et al. (2003) with Chandra. They obtained the column density of $(3.2-4.5) \times 10^{22} \mathrm{~cm}^{-2}$ to the remnant, which is compatible with what we obtained for the pulsar counterpart candidate. The SNR X-ray radiation is dominated by synchrotron emission from an expanding shell. It exhibits a clumpy morphology with an overall elliptical shape with the NE major axis orientation. The radio and Xray emission regions are not correlated (Bamba et al. 2001), which is typical for mixed morphology SNRs. The distance to the remnant and its age were recently estimated to be $9.6 \pm 0.3 \mathrm{kpc}$ and $19 \mathrm{kyr}$, respectively (Ranasinghe \& Leahy 2017).

No pulsar detection has been reported within the remnant, while PSR J1844-0346 is located near its NE boundary which has not been observed by Chandra. The association with the SNR implies the pulsar proper motion $\mu=$

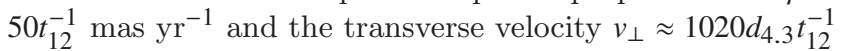
$\mathrm{km} \mathrm{s}^{-1}$, where $t_{12}$ is the pulsar age scaled by its characteristic age of $12 \mathrm{kyr}$ (Table 2 ). This velocity is close to the highest firmly established NS velocity of $\approx 1100 \mathrm{~km} \mathrm{~s}^{-1}$ (Chatterjee et al. 2005). If the real age and distance of the presumed PSR+SNR system are equal to those estimated 
for the remnant, the transverse velocity becomes higher, $1440 \pm 50 \mathrm{~km} \mathrm{~s}^{-1}$, but it is still not outstanding.

\subsection{PSR J2017+3625}

This is the oldest and least energetic but likely closest pulsar in our sample (Table 2). Gotthelf et al. (2016) failed to identify it in X-rays in the same Chandra data-set using the pulsar coordinates from the 3FGL catalogue, which are different from more accurate coordinates obtained from the timing analysis by Clark et al. (2017). There are 10 ACIS source counts from the counterpart candidate detected during the $\approx 10 \mathrm{ks}$ exposure in the $0.3-10 \mathrm{keV}$ range.

We have estimated $N_{\mathrm{H}}$ to the pulsar using the interstellar extinction-distance relation (Green et al. 2015) and the pulsar pseudo-distance of $0.656 \mathrm{kpc}$ (Table 2). The resulting $N_{\mathrm{H}}$ is about $5.3 \times 10^{20} \mathrm{~cm}^{-2}$ which was then frozen in spectral fits. The spectrum was fitted by the PL and BB models.

For the $\mathrm{BB}$ model we have obtained the temperature of about $1 \mathrm{keV}$ which is extremely high for a thermally emitting isolated NS, particularly for this 2 Myr old pulsar. We have thus rejected this model. For the PL model the fit is acceptable, $C$ (d.o.f.) $=7.7(8)$, and it results in $\Gamma=1.2 \pm 0.6$. This is consistent with the spectral slopes of the nonthermal X-ray emission detected in other pulsars. The observed and unabsorbed fluxes in the $0.3-10 \mathrm{keV}$ energy range were $2.2_{-0.8}^{+1.4} \times 10^{-14}$ and $2.3_{-0.8}^{+1.3} \times 10^{-14} \mathrm{erg} \mathrm{cm}^{-2} \mathrm{~s}^{-1}$, respectively. The latter translates in the X-ray luminosity $L_{\mathrm{X}}=1.2_{-0.4}^{+0.7} \times 10^{30} d_{0.656}^{2} \mathrm{erg} \mathrm{s}^{-1}$. The corresponding X-ray efficiency $\log \left(L_{X} / \dot{E}\right) \approx-4$. The ratio $\log \left(G_{100} / F_{X}\right) \approx 3.45$ is consistent with the value of $3.5 \pm 0.5$ derived for the radioquiet $\gamma$-ray pulsars (Abdo et al. 2013).

The detected Chandra source appears to be a plausible $\mathrm{X}$-ray counterpart of this rather old pulsar.

\section{POSSIBLE OPTICAL COUNTERPARTS TO THE X-RAY SOURCES}

The X-ray count statistic is too low for five of the six counterpart candidates to confirm the positional association with gamma-ray pulsars through their spectral properties. Therefore, it is a good idea to check if they coincide positionally with unrelated background optical objects which could emit in X-rays and thus lead to a false pulsar identification. To do that, we have used the Swift Ultraviolet/Optical Telescope (UVOT) images and the results of deep, up to a $\approx 23$ visual magnitude limit, optical sky surveys including the pulsar fields. Specifically, we have considered the recent optical databases of the Panoramic Survey Telescope and Rapid Response System (Pan-STARRS; Flewelling et al. 2016) ${ }^{11}$, and VST Photometric $\mathrm{H} \alpha$ Survey of the Southern Galactic Plane and Bulge (VPHAS+; Drew et al. 2014) ${ }^{12}$.

We have not found any optical objects down to $\approx 23.3$ visual magnitude limit within the position error ellipse of the Chandra counterpart candidate for J2017+3625 (Table 1). This favours the correct X-ray identification of the pulsar.

11 https://panstarrs.stsci.edu/

12 http://www.eso.org/sci/observing/PublicSurveys.html
Within the position error ellipse of the Swift counterpart candidate for J1844-0346 with purely nonthermal Xray spectrum (Sect. 3.5), we have found a relatively bright optical source PSO J184433.074-034628.369 with magnitudes $g=19.729, r=18.169, i=17.243, z=16.715$. By its colour, it appears to be an M-class main sequence star which can hardly produce such a hard X-ray spectrum as is observed for the candidate. We have also not found any known active galactic nuclei at our candidate position which could be responsible for the nonthermal X-ray spectrum.

We have found a relatively bright background star VPHAS J110500.49-603713.41 with $u^{\prime}=17.96, g^{\prime}=16.97$, $r^{\prime}=16.33, i^{\prime}=16.1$ within the position error ellipse of the J1105-6037 Swift X-ray counterpart candidate. Its spectral energy distribution is typical for main-sequence stars.

There are two faint red point objects PSO J055404.767+310739.519 with $i=21.655$ and PSO J055405.198+310742.124 with $i=21.918$ within the position ellipse of the Swift counterpart candidate for J0554+3107. They are not detected in other optical bands. In the absence of any information on the object colours, their nature remains unknown.

Finally, within the position error ellipse of the Swift counterpart candidate for J0002+6216 there are two relatively bright background point optical sources PSO J000257.781+621607.150 with $g=18.9, r=17.9, i=17.42$, $z=17.17$ and $y=17$, and PSO J000257.830+621613.565 with $g=17.76, r=16.9, i=16.49, z=16.29$ and $y=16.1$. Both sources are likely main sequence stars.

Therefore, the X-ray identification of the latter three pulsars has to be viewed with caution. More sensitive, high spatial and temporal resolution X-ray observations can help to distinguish possible false identifications from the real ones.

\section{DISCUSSION AND CONCLUSIONS}

We have found possible X-ray counterparts for six recently discovered $\gamma$-ray pulsars and have proposed SNR associations for two of them. All the counterpart candidates coincide by position well with the pulsars. They are located in the Galactic plane and their X-ray fluxes are $\geq 2 \times 10^{-14} \mathrm{erg}$ $\mathrm{cm}^{-2} \mathrm{~s}^{-1}$. According to the LogN-LogS distribution for the Chandra Galactic Plane sources from Ebisawa et al. (2005), the probability of chance detection of an unrelated X-ray source with $F_{\mathrm{X}}>2 \times 10^{-14} \mathrm{erg} \mathrm{cm}^{-2} \mathrm{~s}^{-1}$ at the $\gamma$-ray pulsar position is $\lesssim 10^{-4}$. This small value supports the correct $\mathrm{X}$-ray identifications.

Despite a low count statistics for most candidates, qualitative conclusions on X-ray spectral properties can be estimated for all of them. These properties are consistent with those observed for other rotation powered pulsars, which demonstrate either a non-thermal PL spectra of the NS magnetosphere origin or a thermal spectra from the NS surface, or a combination of the two. The estimated spectral indices and temperatures are in a good agreement with those observed for other pulsars with similar parameters. Moreover, the ratios of the $\gamma$-ray fluxes to the non-thermal X-ray fluxes, in cases where it is possible to estimate the latter, are also compatible with the respective ratios for other $\gamma$-ray pulsars 
firmly identified in X-rays. Thus, the inferred X-ray properties also favour the correct identification.

The most secure X-ray identification is for PSR J0359+5414 (Sect. 3.2). Almost 500 ks of Chandra observations allowed us to reveal also the diffuse emission around the pulsar. In view of its extent and non-thermal spectrum, it is consistent with PWN emission which can be powered by this quite energetic pulsar. The pulsar counterpart spectrum shows a thermal component from the NS surface, dominating at low photon energies, and a high-energy nonthermal tail of the NS magnetosphere origin, as is observed for Velalike and middle-aged pulsars. Overall, there is little doubt that the Chandra source CXOGSC J035926.0+541455 is the real pulsar counterpart. The absorbing column density toward the pulsar derived from the X-ray spectral fits together with the available extinction-distance relation allowed us to estimate the distance to the pulsar as about $3.45 \mathrm{kpc}$. It is consistent with the $\gamma$-ray pseudo-distance value. The object is a very faint $\mathrm{X}$-ray source. With current X-ray instruments only a mega-second dedicated exposure can help to better constrain its spectral parameters and to find pulsations with the pulsar period.

Medium exposure observations of the other five pulsar counterpart candidates with Chandra and XMM-Newton are necessary to confirm firmly their NS nature. Improved count statistics and detection of pulsations will help to exclude their chance coincidence with background optical stars possibly emitting in X-rays. The suggested associations of PSRs J0002+6216 and J1844-0346 with the SNRs CTB 1 and G28.6-0.1, respectively, can be confirmed by measuring the pulsar's proper motion using a set of Chandra observations separated by a few years. Detection of bow-shock PWNe with tails pointing to the centres of the respective remnants would also favour the associations. In this case, the absorbing column densities to the pulsars derived from the advanced data have to be consistent with those of the SNRs.

After this paper submission, the work by $\mathrm{Wu}$ et al. (2017) appeared where authors using the same archival Xray data briefly reported on five of the six X-ray counterpart candidates thoroughly considered here. However, they presented only a simplified spectral analysis using a single PL model for all the candidates and used only about 10 per cent of the Chandra data available for PSR J0359+5414. The counterpart candidate for PSR J0554+3107 and possible pulsar associations with SNRs are not considered in that paper. The authors have not reported the background optical objects within the X-ray position error ellipses, possibly emitting in X-rays, which may call into question the correctness of the identifications, and have not estimated the probability of chance detection of unrelated X-ray sources at the pulsar's positions. At the same time, they have reported the detection of PSR J0002+6216 in the radio, which is predicted by our X-ray analysis (Sect. 3.1).

\section{ACKNOWLEDGEMENTS}

We are grateful to the anonymous referee for useful suggestions improving the paper and to D.A. Baiko for careful reading the manuscript and valuable comments. The work was supported by the Russian Science Foundation, grant
14-12-00316. It made use of data supplied by the UK Swift Science Data Centre at the University of Leicester, and data and/or software provided by the High Energy Astrophysics Science Archive Research Center (HEASARC), which is a service of the Astrophysics Science Division at NASA/GSFC and the High Energy Astrophysics Division of the Smithsonian Astrophysical Observatory. Based on observations made with ESO Telescopes at the La Silla or Paranal Observatories under programme ID(s) 177.D-3023(B), 177.D3023(C), 177.D-3023(D), 177.D-3023(E).

\section{REFERENCES}

Abdo A. A., et al., 2013, ApJS, 208, 17

Acero F., et al., 2015, ApJS, 218, 23

Allen B., et al., 2013, ApJ, 773, 91

Bamba A., Ueno M., Koyama K., Yamauchi S., 2001, PASJ, 53, L21

Cash W., 1979, ApJ, 228, 939

Chatterjee S., et al., 2005, ApJ, 630, L61

Clark C. J., et al., 2015, ApJ, 809, L2

Clark C. J., et al., 2016, ApJ, 832, L15

Clark C. J., et al., 2017, ApJ, 834, 106

Drew J. E., et al., 2014, MNRAS, 440, 2036

Drimmel R., Cabrera-Lavers A., López-Corredoira M., 2003, A\&A, 409, 205

Ebisawa K., et al., 2005, ApJ, 635, 214

Evans P. A., et al., 2009, MNRAS, 397, 1177

Evans P. A., et al., 2014, ApJS, 210, 8

Fesen R. A., Winkler F., Rathore Y., Downes R. A., Wallace D., Tweedy R. W., 1997, AJ, 113, 767

Flewelling H. A., et al., 2016, preprint, (arXiv:1612.05243)

Foight D. R., Güver T., Özel F., Slane P. O., 2016, ApJ, 826, 66

Fuerst E., Reich W., 1986, A\&A, 154, 303

Fuller J., Cantiello M., Lecoanet D., Quataert E., 2015, ApJ, 810,101

Gotthelf E. V., et al., 2016, ApJ, 826, 25

Green G. M., et al., 2015, ApJ, 810, 25

Hailey C. J., Craig W. W., 1994, ApJ, 434, 635

Halpern J. P., et al., 2014, ApJ, 795, L27

Harrison P. A., Lyne A. G., Anderson B., 1993, MNRAS, 261, 113

Helfand D. J., Becker R. H., White R. L., Fallon A., Tuttle S., 2006, AJ, 131, 2525

Ho W. C. G., Potekhin A. Y., Chabrier G., 2008, ApJS, 178, 102

Hobbs G., Lorimer D. R., Lyne A. G., Kramer M., 2005, MNRAS, 360,974

Kargaltsev O., Pavlov G. G., 2008, in Bassa C., Wang Z., Cumming A., Kaspi V. M., eds, American Institute of Physics Conference Series Vol. 983, 40 Years of Pulsars: Millisecond Pulsars, Magnetars and More. pp 171-185 (arXiv:0801.2602), doi:10.1063/1.2900138

Karpova A., Shternin P., Zyuzin D., Danilenko A., Shibanov Y., 2017, MNRAS, 466, 1757

Koo B.-C., Heiles C., 1991, ApJ, 382, 204

Kramer M., Lyne A. G., Hobbs G., Löhmer O., Carr P., Jordan C., Wolszczan A., 2003, ApJ, 593, L31

Marelli M., De Luca A., Caraveo P., 2013, Mem. Soc. Astron. Italiana, 84, 568

Marelli M., Mignani R. P., De Luca A., Saz Parkinson P. M., Salvetti D., Den Hartog P. R., Wolff M. T., 2015, ApJ, 802, 78

Milne D. K., 1979, Australian Journal of Physics, 32, 83

Noutsos A., Schnitzeler D. H. F. M., Keane E. F., Kramer M., Johnston S., 2013, MNRAS, 430, 2281

Pannuti T. G., Rho J., Borkowski K. J., Cameron P. B., 2010a, AJ, 140, 1787 
Pannuti T., Rho J., Borkowski K., Heinke C., 2010b, in American Astronomical Society Meeting Abstracts \#216. p. 428.15

Pletsch H. J., et al., 2013, ApJ, 779, L11

Popov S. B., Turolla R., 2012, Ap\&SS, 341, 457

Ranasinghe S., Leahy D. A., 2017, preprint, (arXiv:1712.04423)

Rengelink R. B., Tang Y., de Bruyn A. G., Miley G. K., Bremer M. N., Roettgering H. J. A., Bremer M. A. R., 1997, A\&AS, 124,259

Saz Parkinson P. M., et al., 2010, ApJ, 725, 571

Sturrock P. A., 1971, ApJ, 164, 529

Ueno M., Bamba A., Koyama K., Ebisawa K., 2003, ApJ, 588, 338

Wang S., Liu J., Qiu Y., Bai Y., Yang H., Guo J., Zhang P., 2016, ApJS, 224, 40

Watters K. P., Romani R. W., 2011, ApJ, 727, 123

Wongwathanarat A., Janka H.-T., Müller E., 2013, A\&A, $552, \mathrm{~A} 126$

Wu J., et al., 2017, preprint, (arXiv:1712.05395)

This paper has been typeset from a TEX/LATEX file prepared by the author. 